\title{
Evidence-Based Medicine: Systematic Reviews are the Best Evidence Tools for the Practice of Medicine and Dentistry
}

\author{
Fazal Ghani
}

\begin{abstract}
This review presentation first introduces the concepts and approaches necessary for the practice of Evidence-based Medicine (EBM). It then gives details about the importance, need, usefulness and impacts of the systematic reviews (SR) as tools for the practice of EBM. Ways to involve in preparing SR have also been mentioned. The aim is to inform, guide and motivate the prospective clinicians, researchers and academics toward the EBM agenda and to acquaint those concerned and interested in the subject about the conduct of SR. Despite the many obvious advantages of the EBM practice process, the concern of the critics of EBM has also been mentioned. This has been done by reviewing the identified relevant literature on the various aspects and issues of the topic.
\end{abstract}

KEY WORDS: Evidence-based Medicine. Systematic Reviews. Medical and Dental Practice.

\section{INTRODUCTION}

Current trend in the provision of health-care interventions is that they carry evidence for success and efficacy. This is now on the top agenda of all health-care stakeholders and authorities. This evidence, for improved health-care practices, is provided by integrating best clinical evidence, clinical judgment, patient values and circumstances. In order to find the relevant evidence for particular health-care practices, treating physicians are required to be well-versed about the available evidence in the published literature. Unfortunately, health-care practitioners are too busy to find time for this search. Furthermore, the numbers of clinical trials that are published annually in each clinical specialty as well as the number of medical and dental journals are more than too many. In fact many articles per day in each specialty and on each aspect of each specialty are being published in these. For them to stay current, they have to identify, acquire, read, appraise and implement at least one article on daily basis. Even this much is certainly an impossible task. For this purpose, various expert groups and organizations have come forward with a common goal to simplify this task by providing the health-care practitioners and authorities, the best evidence that they distil from the available literature.

\section{FINDING THE EVIDENCE}

The finding of required evidence is a complex process that needs a systematic approach carried out in 5steps. In the first step, a clear question, based on the clinical problem and the expected outcome, has to be generated. This will prompt search for the best evidence. For this, a search strategy has to be done that would assess the literature through various sources that are designed to provide the evidence. The searched and identified literature will then need to be critically evaluated (appraised) for validity, clinical outcome and clinical importance. Subsequently, upon finding appropriate evidence that is relevant to a clinical situation will require an action to be taken to implement it. Finally, it has to be determined whether it would improve the outcome of health-care practices in patients. Asking questions for probing both the background and foreground knowledge related to a clinical situation is important. ${ }^{1}$ The practitioner must be knowledgeable about how specific clinical situations could be managed or prevented (foreground) as well as they must have ascertained about their etiologies. In the process of looking for appropriate evidence, nothing could be more important than asking a focused 4-part question having an acronym PICO, where "P" stands for problem, "I" for intervention, "C" for comparison and "O" for outcome. This could be simply explained by a statement, "In adult patients suffering from amoebic dysentery (problem), would the oral administration of Metronidazole (400mg) tablets twice a day (intervention) when compared to other anti-amoebic tablets (comparison) would result in quicker recovery (outcome)". The best evidence for health-care interventions and practices with their levels of evidence is located in several places. These include the following:

Organizations and Centres Publishing Systematic Reviews and Guidelines:

Cochrane Collaboration (Different Languages) ${ }_{3}^{2}$

Centre for Reviews and Dissemination (CRD). ${ }^{3}$

Scottish Intercollegiate Guidelines Network (SIGN). ${ }^{4}$

National Library for Health (Relevant Specialty). ${ }^{5}$

Clinical Evidence Websites. ${ }^{6}$ 


\section{High Quality Clinical Trials:}

Virtual Health Library (Relevant Languages). ${ }^{7}$

Evidence Based Journals (Relevant Specialty). ${ }^{8}$.

All Clinical Trials: Virtual Health Library ${ }^{9}$ and MEDLINE. ${ }^{10}$

Specialty related Websites for Evidence. ${ }^{11}$

While appraising a particular study for the evidence, the relevant evidence has to fulfill the two important criteria including the Level of the Evidence and the Clinical Utility of the Evidence. ${ }^{11,12}$ The Level of Evidence is the ability of the evidence to reliably predict cause-effect and it is under the influence of the Type of Research Trial or Study. For example, Systematic Reviews (SRs) of Randomized Controlled Trials (RCTs) and the RCTs themselves will generally provide the Highest Level (Level-1) evidence in the Hierarchy of Evidence. Level 2 Evidence that is next in the Hierarchy of Evidence will be provided by the Systematic Reviews of Cohort Trials and by the Cohort Trials themselves. Level-3 Evidence that is inferior to Level2 Evidence will be provided by the Systematic Reviews of Case Control Trials (CCTs). The studies described as Case Series (CSs) and Narrative Reviews (NRs) will provide evidence of Level 4 and Level 5 respectively. Other types of studies such as Case Reports, Epidemiology, Animal Studies and In-Vitro Studies usually are considered extremely poor for providing evidence. Once the Level of Evidence has been established from the type of study, the next step is to determine the Clinical Utility of the Evidence. The Clinical Utility of an Evidence is determined by considering three parameters of the Evidence; first to know about its Validity (Freedom from Bias), second to know about its Importance (Difference between the Experimental and the Control Group) and last but not the least to know about its Clinical Relevance (Importance to Patients - one Treats). With the establishment of the Level and Clinical Utility of Evidence, the next step is to act upon the Evidence. The Goal, obviously, is to implement the best clinical evidence, and using clinical judgment to determine whether the implementation of the evidence will improve care in a practice setting. ${ }^{13}$ Acting upon evidence is a 4-step process involving the stages of Planning, Doing, Studying and Acting. Prior to acting on the evidence, the following 3 key questions must be asked: ${ }^{14}$

What are we trying to accomplish?

What changes can we make?

How will we know that this is an improvement?

\section{SYSTEMATIC REVIEWS}

From the above description of need, steps, processes and tools of evidence for health-care practices, we have learnt that SR are considered as the best tools for providing evidence for health-care practices and interventions. In response to the ever increasing interest in taking health-care decision that are based on evidence, various health professional groups have made the topic of Evidence Based Medical including Dental Practice a global phenomenon taking place at a variety of speeds in different countries. ${ }^{14,15}$ With the idea already widely welcomed by all, the scope of the practice of Evidence-based Medicine (EBM) has become very broad. This can be seen from the everincreasing collaboration between individuals and organizations in their effort to exploit their potential for improved patient's care. ${ }^{16-21}$ Now we see an increased number of RCTs, increased number of systematic reviews, increased links between researchers and organizations, increased number of position statements, development of clinical practice guidelines and the increased need, especially in UK, by the National Health Service (NHS), for the modernization of medical care. ${ }^{14,16,22-29}$ The foundation for the practice of EBM starts with the critical appraisal of primary research. The findings of relevant primary research trials are synthesized in SR. The evidence or otherwise provided by SR is effectively disseminated by researchers, policy-makers, professional and medical care organizations and industry along-with the incorporation and implementation of the findings in clinical practice and undergraduate, postgraduate and professional development education. ${ }^{17,18,21,25,26,30}$ The overall benefit of this process is to facilitate knowledgeable practitioners empowered to provide improved care to their patients. However, the process does not end with this. This is because the evidence and information present is to be put to further objective scrutiny that will provide a feedback to the agenda of new primary research trials. In systematic reviews, the strength of evidence is established across a number of studies, research teams, designs and countries. This is done by a systematic synthesis, using robust and comparable analyses of the findings. Ideally, in systematic reviews, a number of RCTs are reviewed. ${ }^{17,27,28,30,31}$ Then a rigorous, composite overview of the degree of success of a particular intervention or treatment is made through a meta-analysis. ${ }^{16,22,23,27,33,34} \mathrm{~A}$ systematic review being very difficult and exhaustive research work by itself should never be done to later appear as a duplicate effort. In order to avoid this, it is necessary to seek help of international authorities of EBM who can help in this regard. (Table I). Usually, the quality and reliability of systematic reviews is considered good if led by organization like Cochrane Collaboration. ${ }^{21}$ The Cochrane Collaboration is an international organization helping people make informed decisions about health-care services by preparing, maintaining and promoting the accessibility of systematic reviews and the effects of health-care interven- 
tions. Through systematic reviews, evidence based clinical guidelines are drawn. ${ }^{18,19,20,35}$ The evidence available through these efforts may be found through various sources of EBM as mentioned above.

\section{IMPORTANCE, IMPACT, USEFULNESS AND NEED FOR SYSTEMATIC REVIEWS}

A systematic review is a review in which "EVIDENCE" for a health question / problem / issue has been systematically identified, appraised and summarized according to specified criteria. SR provide information about the effectiveness of interventions by identifying, appraising and summarizing the otherwise unmanageable quantities of research. SR may or may not use statistical analyses (meta-analyses). Bias is minimized by using replicable, scientific and transparent approach. SR generate balanced inferences that are based on a coalition and analysis of the available evidence and yet not reflecting the views of experts. They inform policy-making and help decision making about organization and delivery of health and social care. They clarify uncertainty regarding the potential benefit and harm of an intervention and provide empirical answers to focused questions by locating and synthesizing evidence from primary research studies (PRS). Finally, they help us to identify what we know and what we need to know and thus help in planning new research.

While a detailed description for conducting a good quality SR on treatment effectiveness of interventions is beyond the scope of this writing (but available on: www.york.ac.uk/inst/crd/report4.htm), it can be accomplished by following the three stages of Planning Research Review, the actual stage of conducting the SR and the stage of SR Reporting and Dissemination. The Stage of Review Planning requires the consideration of the need for SR, preparation of a proposal for $\mathrm{SR}$, and the development of a review protocol. In the second stage that is in fact actually the SR conduction stage, first the published (or unpublished) research needs to be identified to select the needed research trials. The quality of the so identified research is then assessed for quality so as to proceed to next stages of data extraction, data monitoring and data syntheses. In the last stage, a report is to be written along-with recommendation including practice point(s). This last stage will only be considered completed if the findings of SR are disseminated into practice through its publication and CPD activities at least. Most important to conducting a good quality SR requires the QUOROM Checklist (Published in Lancet 1999; 354: 1896 1899) and the CONSORT Statement Guidelines
(Lancet 2001; 357: 1191 1194). The recommended approach for the critical appraisal of research trials should also be followed (Guyatt, et al 1993). To summarize this, critical appraisal of trials requires the consideration of three broad issues pertaining to "Validity of Results", the "Nature of Results" and the "Local Applicability of the Results". Each of these broad issues shall be addressed from the levels of responses provided by the Trial to several sub-statements / questions (Table I).

The impact of SR on health-care is considerable in terms of bringing changes in policymaking, in changing patient opinions and even in health-care marketing. In 2003, a SR on the efficacy of electric toothbrushes was conducted by the Cochrane Collaboration's Oral Health Group (CCOHG: http:// www.cochrane-oral.man.ac.uk) which is one of the 51 international non-profit Cochrane-Collaboration Groups and is note-worthy for three reasons. Firstly, it systematically examined more than 30 years of published studies and its findings, on 11 January 2003 which were presented at the Forsyth Conference on Evidence-Based Dentistry (EBD) Research Group. Secondly, an international team used international standards to identify, evaluate, compile, analyze and report data. Surprisingly, the SR findings showed that only one type of electric tooth-brush provided a statistically significant though modest, clinical benefit over manual toothbrushes in reducing dental plaque by $7 \%$ and gingivitis by $17 \%$. Thirdly, the SR findings were not based on trials lasting less than 1-month and those published after 2001. During the next week of the CCOHG's presentation, reports about the electric tooth-brush efficacy came in over 100 media outlets in the US, UK, Australia and Asia. The media venues included; The Washington Post, Wall St. Journal, Reuters, Newsweek, CNN, NPR, ABC, and BBC. This media interest may not be surprising, given that $42 \%$ of US adults and $34 \%$ of US teenagers had already viewed toothbrushes as an invention they could not live without. Soon the Lemelson-MIT Invention Index Survey (2003) listed toothbrushes as devices more important than automobiles, PCs, Cell-Phones, and Microwave Ovens.

As valuable as SR can be, their usefulness depends on several aspects. These include the quality of previously published studies. For example, in the Cochrane SR on toothbrushes, 147 PRS were initially identified. Among these, 118 studies did not meet the review standards and that only 29 studies met them. If the purpose of a clinical research trial is to determine clini- 
TABLE I: CRITICAL APPRAISAL OF TRIAL RESULTS

(A good Trial will prompt "Yes" responses to statements mentioned-below).

\begin{tabular}{|c|c|c|c|c|}
\hline $\begin{array}{l}\text { Trial } \\
\text { Results }\end{array}$ & Statement & Yes & $\begin{array}{l}\text { Can't } \\
\text { Tell }\end{array}$ & No \\
\hline VALIDITY & $\begin{array}{l}\text { Did Trial address a clear focused question and in terms of the: } \\
\text { population studied. } \\
\text { interpretations given. } \\
\text { outcomes considered. } \\
\text { Were patients randomized to therapy: } \\
\text { Were all the trial patients properly accounted for at in conclusion and in } \\
\text { terms of : } \\
\text { Was follow-up complete: } \\
\text { Were patients analyzed in the groups to which they were } \\
\text { randomized: } \\
\text { DETAILED QUESTIONS: Related to actual results of the Trial: } \\
\text { Were patients, health workers and study personnel blind to Therapy and in } \\
\text { terms of } \\
\text { the patients: } \\
\text { the health-workers: } \\
\text { the study personnel: } \\
\text { Were the groups similar at the start of the trial and in terms of Age, Sex } \\
\text { and Social Class: } \\
\text { Aside from experimental intervention, were the groups treated equally: }\end{array}$ & & & \\
\hline NATURE & $\begin{array}{l}\text { How large was the Treatment effect? } \\
\text { What outcomes are measured? } \\
\text { How precise was the estimate of the Therapy effect? } \\
\text { What are its confidence limits? }\end{array}$ & & & \\
\hline $\begin{array}{l}\text { LOCAL } \\
\text { APPLIC- } \\
\text { ABILITY }\end{array}$ & $\begin{array}{l}\text { Can they be applied to the local population. } \\
\text { And that the trial patients are similar to the local populace: } \\
\text { Were clinically important outcomes considered? } \\
\text { and if not does this affect the decision? } \\
\text { Are the benefits worth the harms and costs? } \\
\text { and this is unlikely to be addressed by the trial, But: what do you } \\
\text { think? }\end{array}$ & & & \\
\hline
\end{tabular}

(Modified from Guyatt, et al 1993)

cal effectiveness, then the 118 trials not included because of poor trial design or poor trial reporting represents a significant loss of research effort and resources. This observation of poor trial quality or their reporting is not unique to the SR on toothbrushes but is very common as can be seen from the fact that the 27 out of 29 first completed COHG's SR on diverse topics including; Orthodontic Treatment, Treatment of Decay in Primary Teeth, Fluoride Varnishes, Dental Implants, Dentin Hypersensitivity, Treatment of Problems with Temporo-mandibular Disorders (TMD), Treatment of Pre-cancerous Lesions, and the Oral Care of Children Receiving Cancer Treatment, all gave weak and unreliable evidence. Despite the fact that Oral Health sector, in North America, being a multibillion-dollar industry, even recent trials in USA were failing to adhere to Consolidated Standards of Reporting Trials (CONSORT) guidelines. Since the establishment of the Cochrane Collaboration in 1993, interest in the preparation of $\mathrm{SR}$ is on the rise all-over the world. A MEDLINE search can promptly show that annually some more than 2500 RCTs and some 1000 $\mathrm{SR}$ on diverse dental issues only are reported in dental journals. Evidence not available to a clinical question is the main drive for initiating a new SR. But before this, make sure that none exists on the topic. If one existed, then apply the "Database of Abstracts of Reviews of Effectiveness (DARE)" quality appraisal checklist to decide about replacing an out-dated / poor quality SR. On a chosen title, make sure that the SR is not duplicated, is none-existent and not in progress. (Table II) 


\section{TABLE II: \\ SOURCES AVAILABLE TO ASSESS THE NEED FOR A NEW SYSTEMATIC REVIEW}

\section{The Cochrane Library:}

Cochrane Database of SR (CDSR)

Database of Abstracts of Reviews of Effectiveness (DARE)

HTA Database: www.hta.nhsweb.nhs.uk/

Internet sites and Indexes:

TRIP: www.tripdatabase.com

HSTAT: www.text.nlm.nih.gov/

Appraisal Sites:

ARIF: www.bham.ac.uk/arif/enqscomp.htm

NICE: www.nice.org.uk/nice-web/cat.asp?c=153

SIGN Guidelines:

www.show.scot.nhs.uk/sign/home.htm

General Databases:

MEDLINE, EMBASE, CINAHL, PsycLIT, BIOSIS

Researchers:

Personal contacts with Experts in the field

Research in Progress: National Research Register www.update-software.com/national/nrr-frame.html

Systematic reviews are the cornerstones of evidence based medical and dental practices. ${ }^{41}$ Each SR involves a thorough, unbiased, explicit and systematic process whereby all the evidence to a specific welldefined review question is sought, appraised in terms of quality and relevance. ${ }^{41}$ The utility and quality of the resultant $S R$ is influenced by the review question and literature review conducted. Although, secondary research can also be of value it is however considered of inferior quality than a primary or new research. ${ }^{26,31}$ Recently, it has been emphasized that the value and need for each new research must be determined after comparison with an unbiased comprehensive review of the previous work in that field. Most clinicians having the experience of reading SR would have realized the inherently unpredictable nature of SRs. It may appear very disappointing to practitioners to see that so far very few of the many SRs published have provided very little clarity to them about the evidence they are interested to find for their practices. Most of these SRs have generally suggested the need for more and continued work in the respective fields. This general inability of the SRs in providing the high level of evidence is mainly attributed to the lack of availability of good quality published research. Another problem that has been noted is that even many SRs have not been properly conducted. All this prompts us all to think; what on earth, the health-care scientists, researchers and clinicians have been doing all the time by not proving up to task in their research activities. Despite all the existing uncertainty and inability of the SRs in making things clear, one thing has been now very clear to all of us and that is; to redirect our efforts for designing good quality randomized controlled research trials and for following the recommended protocol for conducting SRs. The fruits of this realization will soon become evident in the form of SRs that will provide high-level evidence for our practices, hopefully by the time when the Cochrane Collaboration shall be celebrating its Silver Jubilee event in 2018. Thus it is clear that the outcome of an SR is certainly dependent on the objective analyses of only those studies that meet the specific inclusion criteria. ${ }^{17,23,24,25,29,30,36,37,38}$ On many occasions and surprisingly for many researchers and policy makers, recognition of these criteria remains unknown before the start of an SR. Another problem that is often involved is that many studies, despite being often cited and popular, unexpectedly, fail to pass the pre-set threshold of methodological quality. Guidelines for conducting, reporting and improvement of the quality of SRs have been laid down by expert groups and organizations and their consideration have been shown to have beneficial effects. ${ }^{17,21,23,30}$ The quality of a SR is adversely affected by many factors with some including: ${ }^{16,22,24,25,27,28,34,36}$

- Use of inadequate search strategies and not mentioning the search method(s) used.

- Inadequate inclusion and exclusion criteria.

- Use of inadequate screening and quality assessment of papers.

- Pooling of data.

- Subjective bias in interpretation of findings.

- The poor-way of reporting SRs.

It takes years, for the research to or not to influence the clinical practice. It is also, now, not sufficient for a scientist to carry out a meticulous study, secure its publication and to hope that clinicians and others will act upon its findings. ${ }^{39}$ For the research findings to gain wide acceptance and to be translated to clinical practice by the majority, it needs to be effectively disseminated, and brought to the notice of the profession. ${ }^{38}$ This is not so simple and easy work. Dissemination of the findings of SRs should not only be effective, systematic and consistent but also be attractive. The mere publication of research findings in a journal is considered an ineffective dissemination. This is because most clinicians are too busy, have no access to reading different journals and that many journals do not have clinically useful information. The authors of quality research work tend to gain popularity among the fellow colleagues by focusing on publishing work in high impact journals that normally are not available to many clinicians. Another problem is that even good quality research when published in a low impact journal is not being trusted and read by many. Usually funds are not available to many individual research- 
ers, and if any were they are not sufficient to facilitate wide and effective dissemination of their research findings to all involved in the practice and patients care. Furthermore, many researchers are even not trained in the marketing skills needed for effective communication of their research. There is a need for joint effort, from the researchers, sponsors and health-service organizations, for a planned dissemination of research findings. This aspect should always be a part of research grant application as well as a condition for funding of research. In fact an effective and sincere coalition among researchers, industry, care-providers and professional organizations is a need of the time. With the establishment of close links between researchers, teachers and professional research associations, the evidence based practice could be promoted by organizing activities including EBM symposia and promotion of more SRs etc. Knowing our existing base for evidence based practice, it is important to identify gap in knowledge areas and to instill funding in to those. Research funding bodies and healthcare providers such as Pakistan Medical Research Council (PMRC), Pakistan Science Foundation (PSF) and the Federal and Provincial Health Ministries must consider only the funding of RCTs and SRs. This is the only way for facilitating the delivery of clinically effective care based on current research evidence. Concurrently, the professional associations need to be aware of the complimentary benefits of the EBM by indulging in high quality research that is directly relevant to practice and care. There is also a need for establishing a close link between medical education and research to redesign and initiate undergraduate, postgraduate and professional development programmes so that teaching is done effectively and ethically. Only these can best ensure preparing the "Doctors of Tomorrow". These "Tomorrow's Doctors" will be required to understand both the basic and applied science, able to manage the uncertainty and to understand the importance of knowledge development. The young doctors must be willing to remain updated and willing to change clinical procedures over their life-time clinical practice. This, they can easily do when they are taught critical appraisal skills (CAS) and the modern information management skills. Using this foundation, then the postgraduate and professional development education is built to enhance the CAS, skills for reviewing the literature systematically and learning about the ever-evolving and ever-improving nature of the health-care delivery. These goals are only achievable when all of us involved in providing education and training to students are ourselves actively involved in research and have prepared ourselves to be up-to-date and having knowledge that is the most current. The importance of keeping research and clinical practice close to each other has been well recognized by the health authorities in UK, USA and other countries of the developed world and also in some developing countries. Research and development (R\&D) Programmes and practice based research networks (PBRN) have been already established in many countries of the world. Information relevant to EBM is now available in many ways including some of the following:

1. Clinical Governance that ensures and improves clinical standards of the UK National Institute of Clinical Excellence (NICE) and others.

2. Facilitator agencies and organization for improved implementation of research findings into practice.

3. Sifted, sorted, appraised and unbiased information is made readily available to clinicians through evidence based agencies and journals.

4. Clinical-care-pathways (NHS, UK) that are supported and based on best evidence.

5. Linked bibliographic databases (NeLH).

6. CD-ROMS produced by the Cochrane Collaboration.

7. Clinical Guidelines of the Scottish Intercollegiate Guidelines Network (SIGN).

References for these are given in the list of references.

It has become evident that the practice of medicine must be based on scientific evidence. Following this, EBM, through the tremendous effort of its zealous advocates has come to dominate clinical practice during the past decade or more. Doctors are no more allowed to prefer to rely on experience and expert opinions but to practice that is supported by research and statistical evidence. At the same time, doctors have been advised that during their search of the evidence for their practices, they should be fully able to know what is good and bad science. Some, however, view the evidence-based approach which aims to make clinical decisions follow from statistically valid information in the form of SRs, clinical guidelines or algorithms as ill-informed by the realities, complexities and uncertainties of medical practice. $^{40,41}$ They say that a "rigid reliance" on numbers will not meet every need of an individual patient. On one side such numbers are important, but on the other side, there can never be a purely rational or exact mathematical solution to a patient predicament. They disapprove EBM as it is based on medical scientism and that it conditions doctors to function like a "well-programmed computer" that could only be operational within a strict binary framework. ${ }^{40,41}$ This, they argue, will not work because often patients have conditions or rather combinations of conditions that do not easily match the "supposed evidence". Moreover, patients, sometimes, have problems that are not easy to study scientifically. ${ }^{42} \mathrm{~A}$ strict 
requirement for evidence before acting may mean that practitioners will stop thinking, stop evaluating each patient as unique human being, and stop applying their knowledge to the particularities of the person before them. The provision of treatments that are seemingly supported by statistics may not necessarily be appropriate for each and every individual patient. ${ }^{40}$ This kind of view though not so easily acceptable to many is worth considering as precautionary measure.

\section{CONCLUSION}

Good quality primary research in the form of RCTs and others will provide a sound basis for providing evidence when the findings of these are synthesized in SRs. A systematic implementation of standards of excellence, by all stakeholders, is essential if the health-care community seeks to improve health-care as well as health of the population. All-out efforts must need to be made to ensure that standards of excellence are considered by all Health-care stakeholders including Journals (using them as criteria for editorial and review work when publishing research reports), Funding agencies (as strategies for research planning and reviewing of research grant applications), Professional Associations (when preparing position statements),, Researchers (when designing research trials and their reporting), Product Manufacturers (when deciding about research and claims issues) and Third-Party Payers (when compensating cost of Health-care delivery).

\section{REFERENCES}

1. Sackert DL, et al. Center for Evidence Based Medicine. Focusing Clinical Questions? Available at: www.cebm.net (Accessed 16/08/2007).

2. Cochrane Collaboration. Available at: www.cochrane.org

3. Centre for Reviews and Dissemination (CRD). Available at: www.york.ac.uk/inst/crd

4. Scottish Intercollegiate Guidelines Network (SIGN). Available at: www.sign.ac.uk

5. National Library for Health. Relevant Specialist Library Evidence. Available at: www.nlm.nih.gov

6. Clinical Evidence. Available at: www.clinicalevidence.com

7. Evidence Based Journals (Relevant Specialty Journals). For example in Dentistry. Available at: www.nature.com/ebd/journal

8. Virtual Health Library. www.virtualhealthlibrary.org

9. MEDLINE. www.medline.com

10. Evidence Sites (Relevant Specialty). www.cochrane.org

11. Centre for Evidence-Based Medicine, Oxford. Levels of Evidence and Grades of Recommendations. www.cebm.net
12. Critical Appraisal Skills Program, Oxford. Critical Appraisal Tools.. www.phru.nhs.uk/casp/casp.htm

13. Institute for Health Improvement. Percent of patients receiving an annual dental examination (Check-up). www.ihi.org

14. Scientific and Technical Information Network. www.stnweb.fitz-karlsruhe.de

15. Pitts N. Understanding the jigsaw of evidence based dentistry. Part I: Introduction, research and synthesis. Evid Based Dent. 2004; 5: 2 - 4.

16. Slavin B. Best evidence synthesis. An intelligent alternative to meta-analyses. J Clin Epidemiol. 1995; 48: $9-18$.

17. Center for Reviews and Dissemination. York University, England. www.york.ac.uk/inst/crd/ report4.htm

18. National Institutes for Clinical Excellence Guidelines. www.nice.org.uk/nice-web

19. Department of Health, UK. www.DoH.gov.uk/nhs

20. Data of Abstracts and Reviews of Effectiveness. www.agatha.york.ac.uk/darehp.htm

21. Cochrane Library. www.cochrane.dk/cochrane/ handbook/hbook.htm

22. Dialog. www.dialog.com

23. Guyatt D, Sackett D, Sinclair J, Hayward R, Cook D, Cook R. User's guide to the medical literature. IX. A method for grading the health-care recommendations. JAMA. 1995; 274: $1800-04$.

24. Cook DJ, Sackett DL, Spitzer WO. Methodological guidelines for systematic reviews of randomized controlled trials in health-care from the post dam consultation on meta-analyses. J Clin Epidemiol. 1995; 48: $167-71$.

25. Clark M, Oxman M. Section 5: Locating and selecting studies. In Clark $\mathrm{M}$ and Oxman $\mathrm{M}$ (eds). Cochrane Reviewer's Handbook. Oxford. Also available online at: www.cochrane.dk

26. Britton A, McKee M, Black N, McPherson K, Sanderson $\mathrm{C}$, Bain $\mathrm{C}$. Choosing between randomized and non randomized studies. A systematic review. Health Technol Assess 1998; 2: 1 - 24.

27. Clark MJ, Stewart IA. Obtaining data from randomized controlled trials. How much do we need for reliable and informative meta-analyses? BMJ 1994; 309: 1007 - 10.

28. Cook RJ, Sackett DL. The number needed to treat (NNT). A clinical useful measure of Treatment effect. BMJ. 1995; 310: $452-54$.

29. Drunning M, Abi-Aad G, Gilbert D, Gilliam S, Levitt $H$. Experience, evidence and every day practice. London, King's Fund Publishing, 1999.

30. Counsell $\mathrm{C}$. Formulating the question and locating studies for inclusion in systematic reviews. In: Mulrow C, Cook D (eds). Systematic reviews- syntheses of the best evidence for health-care deci- 
sions. Philadelphia PA, American College of Physicians. 1998; Pp $67-79$.

31. Squires BP. Structured abstracts of original research and review articles. Can Med Assoc J. 1990; 143: 619 - 22.

32. Goodman C. Specify inclusion criteria for studies. Swedish Council on Technology Assessment in Health-care. www.sbu.se/sbu-site/report

33. Tramer MR, Reynolds DJ, Moore RA, McQuay MJ. Impact of covert duplicate publication on meta-analyses. A case study. BMJ. 1997; 315: $365-640$.

34. Walter S. Method of reporting statistical results for medical research studies. Am J Epidemiol. 1995; 141: 896 - 906.

35. Scottish Intercollegiate Guidelines Network Guidelines.: www.whow.scot.nhs.uk/sign/home.htm

36. Counsell $\mathrm{C}$. Formulating the question and locating studies for inclusion in systematic reviews. In: Mulrow C, Cook D (eds). Systematic reviews- syn- theses of the best evidence for health-care decisions. Philadelphia PA, American College of Physicians. 1998: Pp. $67-79$.

37. Sackett D, Wennberg J. Choosing the best research design for each question. BMJ 1997; 315: 1636.

38. Evans D, Hains A. Implementing evidence based changes in health-care. Oxford, Radcliff Medical Press, 2000.

39. Pitts N. Understanding the jigsaw of Evidence based dentistry. Part II: Dissemination of research results. Evidence Based Dentistry. 2004; 5:33 35.

40. Groopman J. How Doctors Think? Ist Ed. Houghton Miffin, USA; 2007.

41. Ghani F, Irfan M. Providing evidence for medical and mental health practice through systematic reviews. J Pak Psych Soc. 2006; 3(2): $70-73$.

42. Horton R. What's wrong with doctors? The New York Books of Review, 2007.

AUTHOR AFFILIATION:

\section{Dr. Fazal Ghani}

Head of Department of Prosthodontics

Khyber College of Dentistry

Khyber Medical University

UNIVERSITY CAMPUS

Peshawar 25120, NWFP, Pakistan.

Email: f.ghani@yahoo.co.in 